\title{
How Simple Cells Are Made in a Nonlinear Network Model of the Visual Cortex
}

\author{
D. J. Wielaard, Michael Shelley, David McLaughlin, and Robert Shapley \\ Center for Neural Science and Courant Institute of Mathematical Sciences, New York University, \\ New York, New York 10012
}

\begin{abstract}
Simple cells in the striate cortex respond to visual stimuli in an approximately linear manner, although the LGN input to the striate cortex, and the cortical network itself, are highly nonlinear. Although simple cells are vital for visual perception, there has been no satisfactory explanation of how they are produced in the cortex. To examine this question, we have developed a large-scale neuronal network model of layer $4 \mathrm{C} \alpha$ in $\mathrm{V} 1$ of the macaque cortex that is based on, and constrained by, realistic cortical anatomy and physiology. This paper has two aims: (1) to show that neurons in the model respond like simple cells. (2) To identify how the model generates this linearized response in a nonlinear network. Each neuron in the model receives nonlinear excitation from the lateral geniculate nucleus (LGN). The cells of the model receive strong (nonlinear) lateral inhibition from other neurons in the model cortex. Mathematical analysis
\end{abstract}

of the dependence of membrane potential on synaptic conductances, and computer simulations, reveal that the nonlinearity of corticocortical inhibition cancels the nonlinear excitatory input from the LGN. This interaction produces linearized responses that agree with both extracellular and intracellular measurements. The model correctly accounts for experimental results about the time course of simple cell responses and also generates testable predictions about variation in linearity with position in the cortex, and the effect on the linearity of signal summation, caused by unbalancing the relative strengths of excitation and inhibition pharmacologically or with extrinsic current.

Key words: primary visual cortex; neuronal network model; simple cells; linearity; synaptic inhibition; phase averaging
Neurons in the primary visual cortex are classified as simple or complex, depending on how they respond to visual stimuli. If the response of the cell depends on the stimulus in an approximately linear fashion, the cell is termed "simple"; otherwise, "complex." Specifically, in response to visual stimulation by the temporal modulation of standing grating patterns, the linear-like behavior of simple cells includes: (1) a sensitive dependence on the spatial phase (position) of the grating, (2) very little presence in a neuron's response of nonlinear distortion components such as second (and higher) temporal harmonics. (This is aside from distortions arising from threshold to firing.) The responses of complex cells are very different: (1) they are spatial phase (position)-insensitive, and (2) their responses are predominantly second harmonic.

The linear dependence on visual stimuli of the simple cell might be assumed to be a simple consequence of convergence of excitatory drive from lateral geniculate nucleus (LGN) cells. However, this ignores the nonlinearities of the LGN cells. For example, rectification caused by the spike-firing threshold produces nonlinear distortion of LGN responses for stimulus contrast $>0.2$, that is, even at relatively low contrast (Tolhurst and Dean, 1990; Shapley, 1994). In the numerical simulations of the

Received Nov. 21, 2000; revised March 30, 2001; accepted April 5, 2001.

This work was supported by The Sloan Foundation for the New York University Theoretical Neuroscience Program, National Institutes of Health Grant 2R01EY01472, National Science Foundation Grants DMS-9971813 and DMS-9707494, and a grant from the US-Israel Binational Science Foundation. We thank Russell DeValois and David Ferster for their permissions to reproduce their published data.

Correspondence should be addressed to Michael Shelley, Courant Institute of Mathematical Sciences, 251 Mercer Street, New York, NY 10012. E-mail: shelley@cims.nyu.edu.

Copyright (c) 2001 Society for Neuroscience $0270-6474 / 01 / 215203-09 \$ 15.00 / 0$ model (see below), this nonlinearity is evident in the responses of cortical cells with only LGN excitation. Such responses contain significant nonlinear components. Therefore, it is an open and important question, how can there be simple cells in the visual cortex?

Surprisingly, there has been as yet no explanation, based on known cortical architecture, for the existence of simple cells in the cerebral cortex. Here we offer an answer to this question by studying a large-scale neuronal network model of layer $4 C \alpha$ in macaque primary visual cortex, V1. Our choice of lateral connectivity within this model is motivated not by Hebbian-based ideas of activity-driven correlations (Troyer et al., 1998), but by our interpretation of the anatomical and physiological evidence concerning cortical architecture, which is known better for macaque V1 than for almost any other cortical area. The crucial distinguishing features of the model, derived from biological data, are that the local lateral connectivity is nonspecific and isotropic, and that lateral monosynaptic inhibition acts at shorter length scales than excitation (Fitzpatrick et al., 1985; Lund, 1987; Callaway and Wiser, 1996; Callaway, 1998). In the model, orientation preference is conferred on cortical cells from the convergence of output from many LGN cells (Reid and Alonso, 1995), with that preference laid out in pinwheel patterns (Bonhoeffer and Grinvald, 1991; Blasdel, 1992a,b; Maldonado et al., 1997). In McLaughlin et al. (2000), we show that orientation selectivity of cells in such a model of $4 C \alpha$ is greatly enhanced by lateral corticocortical interactions. Here we show that: (1) neurons in the network model can behave like simple cells; (2) cancellation of nonlinear LGN excitation by corticocortical inhibition causes the linear-like responses of simple cells in this nonlinear network. 


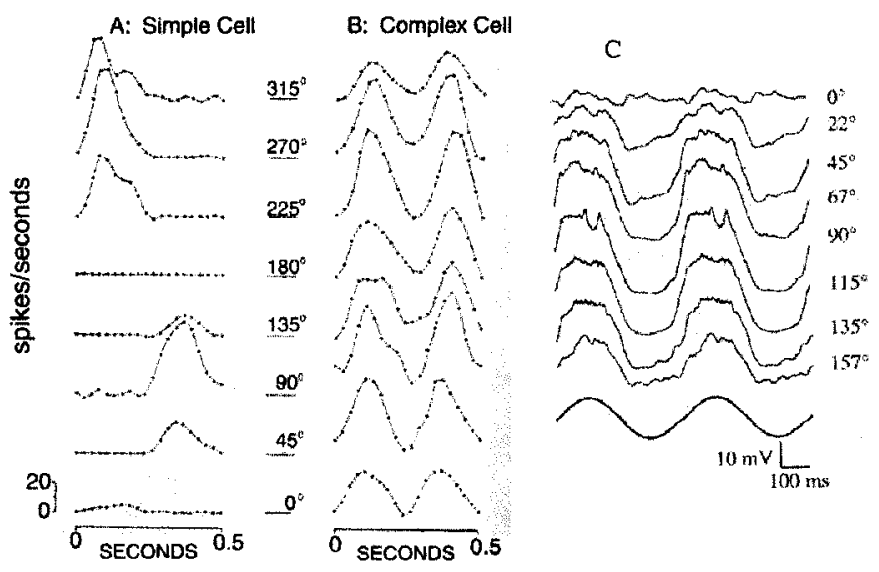

Figure 1. Simple cell responses to grating contrast reversal. $A$ and $B$ are from De Valois et al. (1982) (with author's permission), whereas data in $C$ are from Jagadeesh et al. (1997) (with author's permission). $A$, Macaque monkey simple cell, spike rate response to contrast reversal of a sine grating at $2 \mathrm{~Hz}$ modulation. Position of the standing wave in the visual field is specified in degrees of spatial phase in which one spatial cycle of the grating pattern is $360^{\circ}$. At $\sim 180^{\circ}$, the response goes to zero. $B$, Macaque complex cell response to the same contrast reversal stimulus. The response amplitude shows little variation with spatial phase, and there are two response peaks per cycle of temporal modulation - this is the second harmonic or $F_{2}$ component. $C$, Intracellular responses of a cat simple cell to sine wave contrast reversal at $2 \mathrm{~Hz}$, shown over two cycles. This represents only half a cycle of spatial phase. The temporal modulation waveform is shown below the neural responses. The membrane potential response is predominantly at the fundamental frequency of temporal modulation, with very little modulation at the $0^{\circ}$ phase.

\section{MATERIALS AND METHODS}

\section{Simple cells: experimental classification}

Precise characterization of the linear and nonlinear summation of visual signals of cortical cells was achieved by experiments with drifting and contrast reversal gratings, such as in Movshon et al. (1978), De Valois et al. (1982), and Spitzer and Hochstein (1985), in which experimental techniques that proved useful for studying the linearity of spatial signal summation in retinal ganglion cells (Enroth-Cugell and Robson, 1966; Hochstein and Shapley, 1976) and LGN cells (Kaplan and Shapley, 1982), were applied to visual cortex. Figure $1, A$ and $B$ (De Valois et al., 1982), shows experimental data, based on extracellular recordings of spikes, that illustrate linearity of spatial summation in a simple cell located in macaque V1 (Movshon et al., 1978; Reid et al., 1991). (Note also that simple cell responses are not necessarily linear in all quantities of interest, such as stimulus intensity.) Figure $1 A$ shows the response to contrast reversal stimulation of the cell by a standing pattern at optimal orientation, spatial, and temporal frequency. (Defined more precisely below, "contrast reversal" is the temporal modulation of a standing grating pattern by a sinusoidal modulation of the contrast.) Response to contrast reversal has proven to be a critical test of linearity in simple cells (Spitzer and Hochstein, 1985). The response of the simple cell depends on the spatial phase or position of the standing grating pattern relative to the midpoint of the receptive field of the neuron with a large-amplitude response at the fundamental driving frequency at one spatial phase (the "in-phase" condition) and very little response to "orthogonal phase" stimulation $90^{\circ}$ away. Both responses show little or no generation of the higher temporal harmonics that might be expected for a nonlinear system. However, nonlinear harmonic distortion products are apparent in the responses of cortical complex cells (De Valois et al., 1982), an example of which is shown in Figure $1 B$. Note, in particular, the phase insensitivity and the frequency-doubled (2nd harmonic) response of the complex cell. It is worth noting that the simple cells in De Valois et al. (1982) were not assigned to a cell layer and that subsequent experimental work in recording the activity of neurons across all layers of macaque V1 has found many neurons in layer $4 C \alpha$ that behave just the same as the simple cell illustrated in Figure 1A (Ringach et al., 2001).

There have been some measurements, in the cat visual cortex, of intracellular responses of simple cells to such stimuli (Ferster et al., 1996;
Jagadeesh et al., 1997). Such data are shown in Figure $1 C$, which shows the membrane potential (with spikes filtered) of a cat simple cell responding to contrast reversal stimulation, for in-phase and orthogonal phase spatial patterns. When the stimulus grating is in-phase, there is a large component of the membrane potential that appears to be approximately sinusoidal in time, at the same frequency as the temporal modulation of the stimulus. When the stimulus grating is moved to the orthogonal phase position, the modulation of the membrane potential is small in amplitude, with a very small second harmonic. These results are consistent with the linearity of an extracellular response of a simple cell.

\section{Computational model}

Description. Our model is a large-scale neuronal network of layer $4 C \alpha$, comprised of excitatory and inhibitory integrate-and-fire (I\&F) point neurons. The simulated neurons and the conductance-based interactions in the model are like those used by many others before us. What distinguishes this model is its reliance on cortical architecture to specify the corticocortical connections, and in the choice of connection strengths that yield responses that match physiological data. The architecture of the model derives from cortical anatomy (Fitzpatrick et al., 1985; Callaway and Wiser, 1996; Callaway, 1998) and optical imaging experiments. Optical imaging (Bonhoeffer and Grinvald, 1991; Blasdel, 1992a,b; Maldonado et al., 1997) reveals orientation hypercolumns with "pinwheel" patterns of orientation preference in the superficial layers $2 / 3$ of the cortex; neurons of like-orientation preference reside along the same radial spoke of a pinwheel, with the preferred angle sweeping through $180^{\circ}$ as the center of the pinwheel is encircled. We assume that there are pinwheel patterns in layer $4 C$, parallel to those in layers $2 / 3$. This assumption is based on the classical concept that there are orientation columns in V1 cortex (Hubel and Wiesel, 1962). The orientation preference map is assumed to be hard-wired into the cortex during development, through the orientation preference of each group of LGN cells that converge onto each cortical cell (Reid and Alonso, 1995).

The model [described in more detail in McLaughlin et al. (2000)] is of a small patch of cortex $\left(1 \mathrm{~mm}^{2}\right.$, containing four hypercolumns and four orientation pinwheels) of input layer $4 C \alpha$. It is a conductance-based model that consists of a two-dimensional lattice of $128^{2}$ coupled I\&F neurons, of which $75 \%$ are excitatory and $25 \%$ are inhibitory.

Basic equations of the model. Let $v_{E}^{j}\left(v_{I}^{j}\right)$ be the membrane potentials of excitatory (inhibitory) neurons. In the model, they evolve by the coupled system of differential equations,

$$
\frac{d v_{P}^{j}}{d t}=-\lambda v_{P}^{j}-g_{P E}^{j}(t)\left[v_{P}^{j}-V_{E}\right]-g_{P I}^{j}(t)\left[v_{P}^{j}-V_{I}\right]
$$

where $P=E, I$ and the superscript $j=\left(j_{1}, j_{2}\right)$ indexes the spatial location of the neuron within the cortical layer. We specified the cellular biophysical parameters, using commonly accepted values: the capacitance $C=$ $10^{-6} \mathrm{~F} \mathrm{~cm}{ }^{-2}$, the leakage conductance $g_{R}=50 \times 10^{-6} \Omega^{-1} \mathrm{~cm}^{-2}$, the leakage reversal potential $V_{R}=-70 \mathrm{mV}$, the excitatory reversal potential $V_{E}=0 \mathrm{mV}$, and the inhibitory reversal potential $V_{I}=-80 \mathrm{mV}$. We took the spiking threshold as $-55 \mathrm{mV}$ and the reset potential to be equal to $V_{R}$. The membrane potential and reversal potentials were normalized to set the spiking threshold to unity and the reset potential (and thus $V_{R}$ ) to zero, so that $V_{E}=14 / 3, V_{I}=-2 / 3$, and generally $-2 / 3 \leq v_{E}^{j}, v_{I}^{j} \leq 1$. The capacitance does not appear in Equation 1 because all conductances were redefined to have units of $\sec ^{-1}$ by dividing through by $C$. This was done to emphasize the time scales inherent in the conductances; For instance the leakage time-scale is $\lambda^{-1}=20 \mathrm{msec}$. True conductances are found by multiplication by $C$.

Conductances. The time-dependent conductances arise from the input forcing (through the LGN) and from noise to the layer, as well as from the cortical network activity of the excitatory and inhibitory populations. They have the form:

$$
\begin{gathered}
g_{E E}^{j}(t)=F_{E E}(t)+S_{E E} \sum_{k} a_{j-k} \sum_{l} G_{E}\left(t-t_{l}^{k}\right), \\
g_{E I}^{j}(t)=f_{E I}^{0}(t)+S_{E I} \sum_{k} b_{j-k} \sum_{l} G_{I}\left(t-T_{l}^{k}\right),
\end{gathered}
$$

with similar expressions for $g_{I E}^{j}$ and $g_{I I}^{j}$, and where $F_{P E}(t)=g_{\operatorname{lgn}}^{j}(t)+$ $f_{P E}^{0}(t), P=E, I$. Here $t_{l}^{k}\left(T_{l}^{k}\right)$ denotes the time of the $l$ th spike of the $k$ th excitatory (inhibitory) neuron.

The conductances $f_{P P^{\prime}}^{0}(t)$ are stochastic. Unless stated otherwise, their 
means and SDs were taken as $f_{E E}^{0}=f_{I E}^{0}=6 \pm 6 \mathrm{sec}^{-1}, f_{E I}^{0}=f_{I I}^{0}=85 \pm$ $35 \mathrm{sec}^{-1}$. These conductances have an exponentially decaying autocorrelation function with time constant $4 \mathrm{msec}$. The constant background $g_{0}$ of the LGN drive $g_{\operatorname{lgn}}$ is taken as $35 \mathrm{sec}^{-1}$. The kernels $(a, b, . .)_{k}$ represent the spatial coupling between neurons. Only local cortical interactions (i.e. on scales $<500 \mu \mathrm{m}$ ) are included in the model, and these are assumed to be isotropic (Fitzpatrick et al., 1985; Lund, 1987; Callaway and Wiser, 1996; Callaway, 1998), with Gaussian profiles for the kernels $(a, b, . .)_{k}$. Based on the same anatomical studies, we estimate that the spatial length scale of excitation exceeds that of inhibition and that excitatory radii are of order $200 \mu \mathrm{m}$ and inhibitory radii of order $100 \mu \mathrm{m}$.

The cortical temporal kernels $G_{\sigma}(t)$ model the time course of synaptic conductance changes in response to arriving spikes from the other neurons. They are of the form:

$$
G_{\sigma}=c_{\sigma}^{c} \frac{t^{5}}{\tau_{\sigma}^{6}} \exp \left(-t / \tau_{\sigma}\right) H(t), \sigma=E, I,
$$

where $H(t)$ is the unit step function. The time constants are based on experimental observations (Koch, 1999; Azouz et al., 1997) (A. Reyes, personal communication). The time constant for excitation $\left(\tau_{E}=0.6\right.$ msec; time to peak is $3 \mathrm{msec})$ is shorter than that for inhibition $\left(\tau_{I}=1.0\right.$ msec; time to peak is $5 \mathrm{msec}$ ). In addition, based on recent experimental findings (Gibson et al., 1999), we add a second, longer time-course of inhibition $(\sim 30 \mathrm{msec}$ in duration $)$.

The behavior of the computational model depends on the choice of the corticocortical synaptic coupling coefficients: $S_{E E}, S_{E I}, S_{I E}, S_{I I}$. All cortical kernels have been normalized to unit area. Hence, the coupling coefficients represent the strength of interaction and are treated as adjustable parameters in the model. In the numerical experiments reported here, the strength matrix $\left(S_{E E}, S_{E I}, S_{I E}, S_{I I}\right)$ was set to be $(0.8,9.4$, $1.5,9.4)$. This matrix means that excitatory neurons excite inhibitory neurons almost twice as much as they excite other excitatory neurons but that inhibitory neurons inhibit excitatory neurons and other inhibitory neurons with equal strength. Also, inhibitory neurons have much stronger coupling to all other cortical neurons than do excitatory neurons. We explored many strength matrices in many numerical experiments. If the corticocortical excitation was too strong, oscillations resulted. If the corticocortical inhibition was too weak, the responses of the cells were nonlinear and not selective enough. If inhibition was too strong, the response of the network became too small. The matrix given here generated simple cells that had the orientation selectivity, and the magnitude and dynamics of response, seen in physiological experiments (McLaughlin et al., 2000). This seems contrary to anatomical studies that show V1 cortex has a preponderance of excitatory synapses (Beaulieu et al., 1992). However, the biological cortex is filled with orientationselective cells that are not only simple, but also complex, as well as cells whose responses lie between these classifications. It seems likely that once the sources of this diversity are understood and properly accounted for, the constraints on coupling strengths to produce simple cells will be different and the role of corticocortical excitation will be elucidated.

Contrast reversal stimuli. Let $I(\vec{x}, t)$ be the space- and time-dependent intensity of the visual stimulus. A "contrast reversal" stimulus is given by:

$$
I(\vec{x}, t)=I_{0}[1+\epsilon \sin (\omega t) \cos (\vec{k} \cdot \vec{x}-\phi)],
$$

with parameters $I_{0}$ (intensity), $\epsilon$ (contrast), $\omega$ (temporal frequency), and $\phi$ (phase). The parameter $\vec{k} \equiv k(\cos \theta, \sin \theta)$, where $k$ denotes the spatial frequency and $\theta$ the orientation of the grating pattern. In the computational experiments, we used $k=3$ cycles $/^{\circ}$.

$L G N$ response to contrast reversal stimuli. The total input into the $j$ th cortical neuron arrives from $N(=17)$ LGN cells:

$$
g_{\operatorname{lgn}}^{j}(t)=\sum_{i=1}^{N}\left\{g_{0}^{j}+\int_{0}^{t} d s \int d \vec{x} G_{l g n}(t-s) A\left(\vec{x}_{i}^{j}-\vec{x}\right) I(\vec{x}, s)\right\}^{+} .
$$

Here $\{R\}^{+}=R$ if $R>0 ;\{R\}^{+}=0$ if $R \leq 0 ; g_{0}^{j}$ represents the maintained (background) activity of the LGN neurons feeding into the $j$ th cortical neuron, in the absence of visual stimulation. The summed LGN input, $g_{l g n}^{j}(t)$, into a cortical neuron depends nonlinearly on the visual stimulus $I(\vec{x}, t)$, because of rectification. There may be some additional nonlinear input from the magnocellular Y cells (Kaplan and Shapley, 1982). We have not modeled this group of cells because the percentage of such cells is small $(<25 \%)$ and because the cortical mechanisms we propose will tend to linearize the input of $\mathrm{Y}$ cells to cortex also, so no new principle is involved.

The temporal kernel $G_{l g n}(t)$ and spatial kernel $A(\vec{x})$ of an LGN cell are chosen to agree with experimental measurements (Benardete and Kaplan, 1999) (R. Shapley and R. C. Reid, unpublished observations). Their functional forms are:

$$
\begin{aligned}
G_{l g n}(t) & =c_{0} t^{5}\left[\exp \left(-t / \tau_{0}\right)-c_{1} \exp \left(-t / \tau_{1}\right)\right], \\
A(\vec{y}) & = \pm\left\{\frac{a}{\pi \sigma_{a}^{2}} \exp \left[-\left|\vec{y} / \sigma_{a}\right|^{2}\right]-\frac{b}{\pi \sigma_{b}^{2}} \exp \left[-\left|\vec{y} / \sigma_{b}\right|^{2}\right]\right\},
\end{aligned}
$$

where $\tau_{0}=3 \mathrm{msec}, \tau_{1}=5 \mathrm{msec}, \sigma_{a}=0.066^{\circ}, \sigma_{\mathrm{b}}=0.093^{\circ}, a=1$, and $b=$ 0.74 where + represents an "on-center," and - an "off-center" LGN cell. The constant $c_{1}$ is determined so that the kernel $G(t)$ integrates to zero, as is approximately the case for LGN neurons in the magnocellular pathway.

The spatial arrangement of LGN cell receptive field centers, $\vec{x}_{i}^{j}$, is as segregated on-off subregions (Reid and Alonso, 1995)-here a center subregion of like-polarity cells with twin flanks of opposite polarity. This segregation confers an orientation preference on the input to each cortical cell, and this preference is laid out in pinwheel patterns. Additionally, the center of the receptive field of each cortical cell (created through the aggregate LGN input) is randomized. This was done to account for diversity in the location of this receptive field center and random variations in the spatial symmetry of the on-off subregions. It confers a preferred spatial phase on the LGN input of each cortical cell. From cortical cell to cell this spatial phase preference is distributed randomly over a broad range, as has been found in recent measurements (DeAngelis et al., 1999).

When the stimulus is contrast reversal, Equation 3 for the input conductance simplifies (for $t \gg \tau_{0}$ ) to:

$$
g_{\operatorname{lgn}}^{j}(t)=\sum_{i=1}^{N}\left\{g_{0}^{j}+p_{i}^{j} \sin \left(\omega\left(t-t_{S}\right)\right)\right\}^{+},
$$

where $t_{S}$, the temporal phase shift caused by the retina and LGN, depends only on the choice of $G_{l g n}(t)$ and on the temporal grating frequency $\omega$. An individual term in this sum is simply a rectified sinusoid (if $\left|p_{i}^{j}\right|>g_{0}^{j}$ ), which takes on absolute maxima either at $1 / 4$ cycle $\left(p_{i}^{j}>0\right)$ or at $3 / 4$ cycle $\left(p_{i}^{j}<0\right)$, with respect to $\omega \cdot t_{S}$. For convenience, we set $t_{S}=0$.

\section{RESULTS}

\section{Contrast reversal}

Contrast reversal of a grating pattern is an effective stimulus for classifying cells as simple or complex. In response to contrast reversal, the summed LGN drive in the model has (for 100\% contrast modulation) the generic spatial phase and time dependence shown in Figure 2. Notice that (1) for each phase, the sinusoidal shape is significantly distorted, (2) the absolute maxima occur at either $1 / 4$ cycle or $3 / 4$ cycle, and (3) the orthogonal phase case (with the lowest peak heights of response) possesses two absolute maxima per cycle, resulting in a pronounced frequency doubling. This is produced by the rectification in Equation 4 and occurs in particular when a line of constant luminance ( $I=I_{0}$ in Eq. 2) lies down the middle of a segregated subregion of on-center (or off-center) LGN cells. In this case, the stimulus modulation is elevated first on one side of this line, then on the other, during one temporal period of the stimulus. And so, during one temporal period, the modulation brings to fire first the on-cells on one side of this line, then brings to fire the on-cells on the other side, producing a frequency-doubled aggregate LGN response.

Unlike their LGN input, cortical neurons in the model behave like simple cells in the contrast reversal experiment. Figure $3 a-c$ shows data from an excitatory model neuron located near a pinwheel center. In Figure $3 a$ both the "in-phase" and "orthogonal phase" membrane potential responses are shown. Here, the spike and reset mechanism of this neuron has been turned off- 

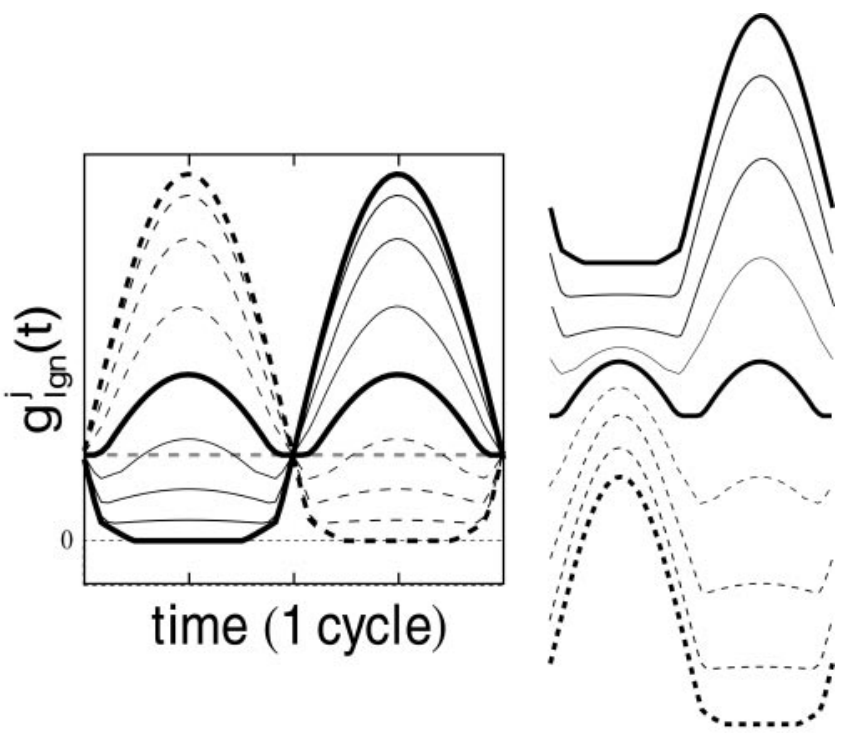

Figure 2. In the model, the conductance received by the $j$ th cortical neuron from the LGN, $g_{l g n}^{j}(t)$ : From contrast reversal gratings (at preferred orientation, $100 \%$ contrast, optimal spatial and temporal frequencies). Responses to nine different grating spatial phases $\left(\phi=\phi_{P}^{i}+i \pi / 8\right.$, $i=0,1,2, \ldots, 8)$ are shown. One thick curve is the maximal "in-phase" case $\left(\phi=\phi_{P}^{j}\right)$; and the second thick curve is the minimal "orthogonal phase" case $\left(\phi=\phi_{P}^{j}+\pi / 2\right)$. For contrast reversal results, the time axis has been translated so that $t=0$ corresponds to the initial arrival of excitations in V1 from the LGN. On the right side of the figure, the different response waveforms have been separated vertically to correspond to the data format of Figure 1.

blocked-so that the waveform of stimulus-modulated potential, $v_{b}$, can be seen more easily and compared with the experimental data (where spikes have been filtered). Thus, the averaged waveforms of the membrane potentials in Figure $3 a$ should be compared with those shown in Figure $1 C$. There is a good degree of similarity. Extracellular spike counts for this same neuron (spike and reset now on) are displayed in Figure $3, b$ and $c$, as cycle averaged histograms, and these are comparable to the simple cell data in Figure $1 A$.

Figure $3 a-c$ shows that the computational model captures the linearity seen experimentally in simple cells. The in-phase response is predominantly at the fundamental driving frequency. The spike rate is not modulated at the second harmonic when the stimulus is at the orthogonal phase, and the membrane potential shows very little second harmonic (or $F_{2}$ ) component in its orthogonal phase response, consistent with experimental measurements.

\section{Removing corticocortical interactions in the model}

To study the effect of lateral corticocortical interactions in the model, we shut them off and observed the consequences. Figure $3 d-f$ shows the results of a simulation with all network interactions shut off but with the LGN and noise terms the same as in the full network simulation shown in Figure $3 a-c$. For the orthogonal phase case, notice the large amplitude of the second harmonic in both the spike rate response and the membrane potential. This second harmonic response is inherited from the LGN input (as seen in Fig. 2 in the orthogonal phase LGN stimuli).

The in-phase response of the uncoupled neuron in Figure $3 d$ has a very large peak spike rate relative to that of the fully coupled network. This result indicates the presence of very strong corticocortical inhibition in the fully coupled network.
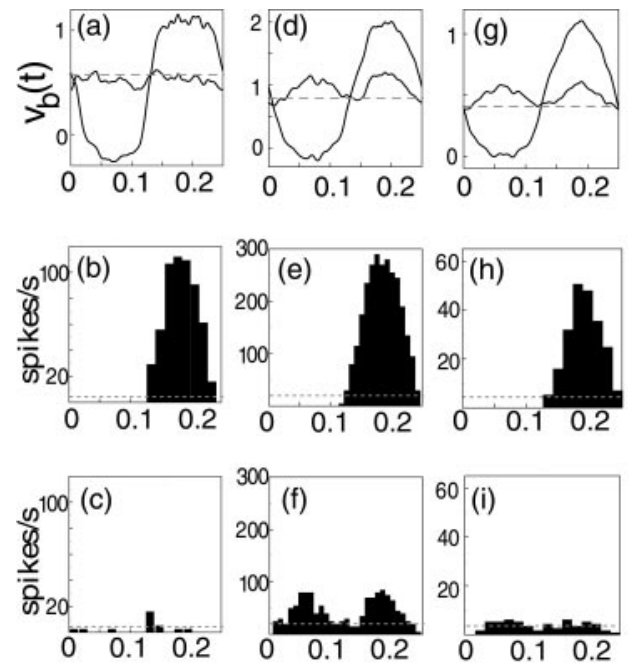

time (sec)

Figure 3. Responses in the model to in-phase and orthogonal phase, 4 $\mathrm{Hz}$ contrast reversal gratings (at $100 \%$ contrast, preferred angle and optimal spatial frequency), for an excitatory neuron near a pinwheel center. The left column $(a-c)$ shows responses for a representative fully coupled neuron, and the middle column $(d-f)$ when this neuron is uncoupled from the network. The right column $(g-i)$ shows responses for a feedforward uncoupled neuron, for which the background mean and noise, and the LGN drive, have been adjusted downward to give spike rates in a normal range. The first row $(a, d, g)$ shows cycle-averaged membrane potentials, with spike and reset blocked. Cycle-averaged spike histograms (when spikes are not blocked) are shown below the membrane potentials [in-phase $(b, e, h)$ and orthogonal phase $(c, f, i)$ ]. The cycle averages were computed over 24 cycles of the stimulus. Dashed horizontal lines are the background responses.

The responses of an uncoupled model neuron are much larger than seen in the living cortex, because of the removal of strong inhibition in the model. Another approach to cortical modeling is to choose different input and internal noise parameters for the uncoupled model neurons to fit the background and peak firing rates of the real cortex. We did this and investigated the responses of what we called a "feedforward" neuron with much weaker LGN drive ( $\left.g_{\text {lgn }}\right)$ and stochastic background $\left(f_{E E}, f_{E I}\right)$ than in the full model. In this case the LGN drive and the means and SDs of the noise were adjusted downward, as follows: now $g_{0}=10 \mathrm{sec}^{-1}$, $f_{E E}^{0}=f_{I E}^{0}=6 \pm 6 \mathrm{sec}^{-1}$, and $f_{E I}^{0}=f_{I I}^{0}=45 \pm 25 \mathrm{sec}^{-1}$. The results of the simulation for the feedforward neuron are shown in Figure $3 g-i$. Compared with both the responses of the feedforward and uncoupled neurons, the membrane potential of the fully coupled neuron has a much smaller $F_{2}$ component, because of corticocortical interactions.

\section{Mechanisms of linearization}

To understand the mechanisms by which the model cortex produces simple cells, we return to the governing equation for the $j$ th cortical excitatory cell, and write it as:

$$
\frac{d v^{j}}{d t}=-g_{T}^{j}(t) v^{j}+I_{D}^{j}(t)
$$

where

$$
\begin{gathered}
g_{T}^{j}(t)=\lambda+g_{l g n}^{j}(t)+g_{E E}^{j}(t)+g_{E I}^{j}(t) \\
I_{D}^{j}(t)=\left(g_{l g n}^{j}(t)+g_{E E}^{j}(t)\right) V_{E}-g_{E I}^{j}(t)\left|V_{I}\right| .
\end{gathered}
$$




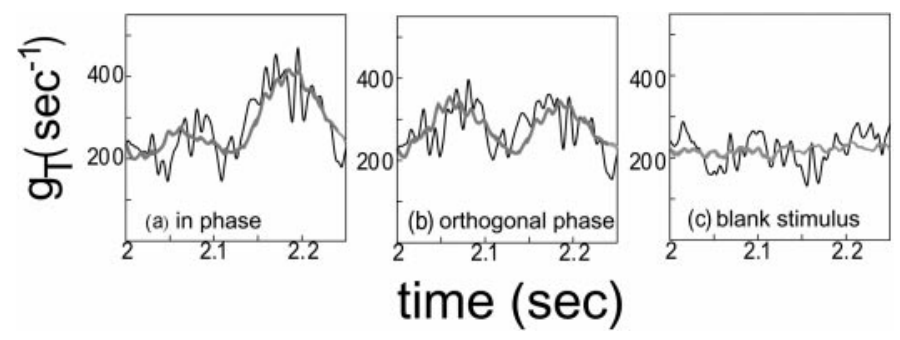

Figure 4. The total conductance for the in-phase stimulus $(a)$, the orthogonal phase stimulus $(b)$, and the blank stimulus $(c)$. The cycle averaged conductance $\bar{g}_{T}$ (averaged over 24 cycles) is shown as a thick gray curve, superimposed on the (less smooth) instantaneous conductance $g_{T}$ over one cycle ( $4 \mathrm{~Hz}$ contrast reversal stimulus). These are simulations for the near neuron in the fully-coupled network in Figure 3.

Here $g_{T}^{j}$ is the total conductance and is the inverse of an effective "integration time" for this neuron. We call $P_{D}$ the difference current, because it is the difference of currents generated by the excitatory and inhibitory conductances. As the membrane potential $v^{j}$ was normalized by the difference between resting and threshold potential, it is dimensionless. Furthermore, having scaled by the fixed membrane capacitance, both the total conductance $g_{T}$ and difference current $I_{D}$ have units of sec ${ }^{-1}$. We now study these two quantities.

\section{The total conductance}

The total conductance $g_{T}$, at in- and orthogonal phases, is shown in Figure 4, $a$ and $b$, as a function of time within the cycle of contrast reversal. This figure is from data for the fully coupled model neuron of Figure $3 a-c$. There are two key points to note: (1) the conductance $g_{T}$ is large, exceeding $400 \mathrm{sec}^{-1}$ under stimulation. The model is working in this high conductance regime to achieve known properties of the biological visual cortex: stability, high responsiveness, and relatively high stimulus selectivity (McLaughlin et al., 2000). Indeed, large conductances have been measured under visual stimulation in the cat visual cortex (Borg-Graham et al., 1998; Hirsch et al., 1998). One can calculate from Equation 5 that the high conductance implies that, when spikes are blocked, the membrane potential $v_{b}^{j}$ is well approximated by:

$$
v_{b}^{j} \approx I_{D}^{j} / g_{T}^{j}
$$

We find numerically that this equality holds in very good approximation for the cycle averaged quantities, that is,

$$
\bar{v}_{b}^{j} \approx \bar{I}_{D}^{j} / \bar{g}_{T}^{j},
$$

where $\bar{f}(t)=N^{-1} \sum_{n=0}^{N-1} f(t+2 \pi n / \omega)$. (Henceforth we drop the overbar, and assume it, unless stated otherwise.) A conclusion from this analysis is that one can understand the behavior of the membrane potential by studying the dependence of $I_{D}$ and $g_{T}$ on the visual stimuli. And (2), as can be seen in Figure 4, the conductance $g_{T}$ has a waveform in response to contrast reversal that is highly distorted and, in particular, contains a large $F_{2}$ component at the orthogonal phase.

Of course the origin of these features lies in the constituent conductances that make up $g_{T}$. These conductances also comprise $I_{D}$, which we now consider.

\section{The difference current}

Plots of the difference current $I_{D}$, together with its constituent currents from Equation 7, are shown in Figure 5. $I_{D}$ and its components are shown at both the in-phase $(a)$ and orthogonal phase $(b)$ for contrast reversal stimulation. The current contributed by the LGN drive is graphed in green, the corticocortical excitatory current is graphed in red, and the corticocortical inhibitory current is graphed in blue. From Equation 7 the difference current $I_{D}$ (graphed in black) is simply the sum of these three currents.

Again, there are key points to note: (1) Figure $5 b$ shows that corticocortical currents, whether at in- or orthogonal phase, have primarily second harmonic distortions, with inhibitory corticocortical currents significantly larger than excitatory. (2) By comparing Figure 5, $a$ and $b$, it is clear that the corticocortical currents are mostly insensitive to the spatial phase of the grating. And (3), it is consequently only the LGN drive that provides the large modulation at the first harmonic in both $g_{T}$ and $I_{D}$ for the in-phase stimulus.

It is interesting to compare the components of the conductance for the contrast reversal experiment at orthogonal spatial phase. This is displayed in Figure 6. There it can be seen that the corticocortical inhibitory conductance of the model is the predominant component. This figure also shows that the inhibitory conductance is stronger for neurons far from the pinwheel singularities.

What underlies the absence of modulation of the membrane potential $v_{b}$ at orthogonal phase? Recall that at orthogonal phase, the total conductance $g_{T}$ is modulated at $F_{2}$. This modulation is in phase with the $F_{2}$ modulation of the difference current $I_{D}$, as is evident in Figure $5 b$. Then $v_{b} \approx I_{D} / g_{T}$ is approximately constant in time because $I_{D}$ and $g_{T}$ are approximately proportional. And the proportionality of $I_{D}$ and $g_{T}$ is partly a consequence of the fact that corticocortical inhibition is the predominant term in $g_{T}$.

Thus, simple-cell intracellular responses occur in the model because its corticocortical conductances have significant $F_{2}$ modulations that cancel the $F_{2}$ coming from the input. But why are such modulations present in the cortex? The reason is that the $j$ th neuron receives spikes from many other cortical neurons, each of which is responding individually in a manner sensitive to the phase of its own LGN drive. This individual phase dependence arises because each of these cortical neurons is driven by LGN excitation, and each summed LGN drive will have its own temporal waveform that will be one of those sketched in Figure 2. The excitation of each LGN cell is maximal at $1 / 4$ or $3 / 4$ temporal cycle. Because the corticocortical input to the $j$ th neuron is an average over many such phase-sensitive responses, some of which peak at $1 / 4$, some at $3 / 4$ cycle, this results in a total corticocortical conductance, which peaks at both the $1 / 4$ and $3 / 4$ temporal cycle, and consequently has significant $F_{2}$ content. In summary, the corticocortical modulations have large, phase-insensitive, $F_{2}$ modulations because the isotropic cortical architecture of the model allows an averaging over the activity of many cortical neurons, and thus, indirectly averages over the many preferred spatial phases of the LGN input [as suggested by the results in DeAngelis et al. (1999)], which peak at $1 / 4$ and 3/4 cycle. This "phase averaging" by the network is similar to that used in a model for complex cells (Chance et al., 1999). It should be emphasized that although we have invoked phase averaging as the mechanism for producing frequency-doubled cortical input, this state of cortical activity arises from the dynamics of the system in a way consistent with its architecture. It is not imposed a priori. 


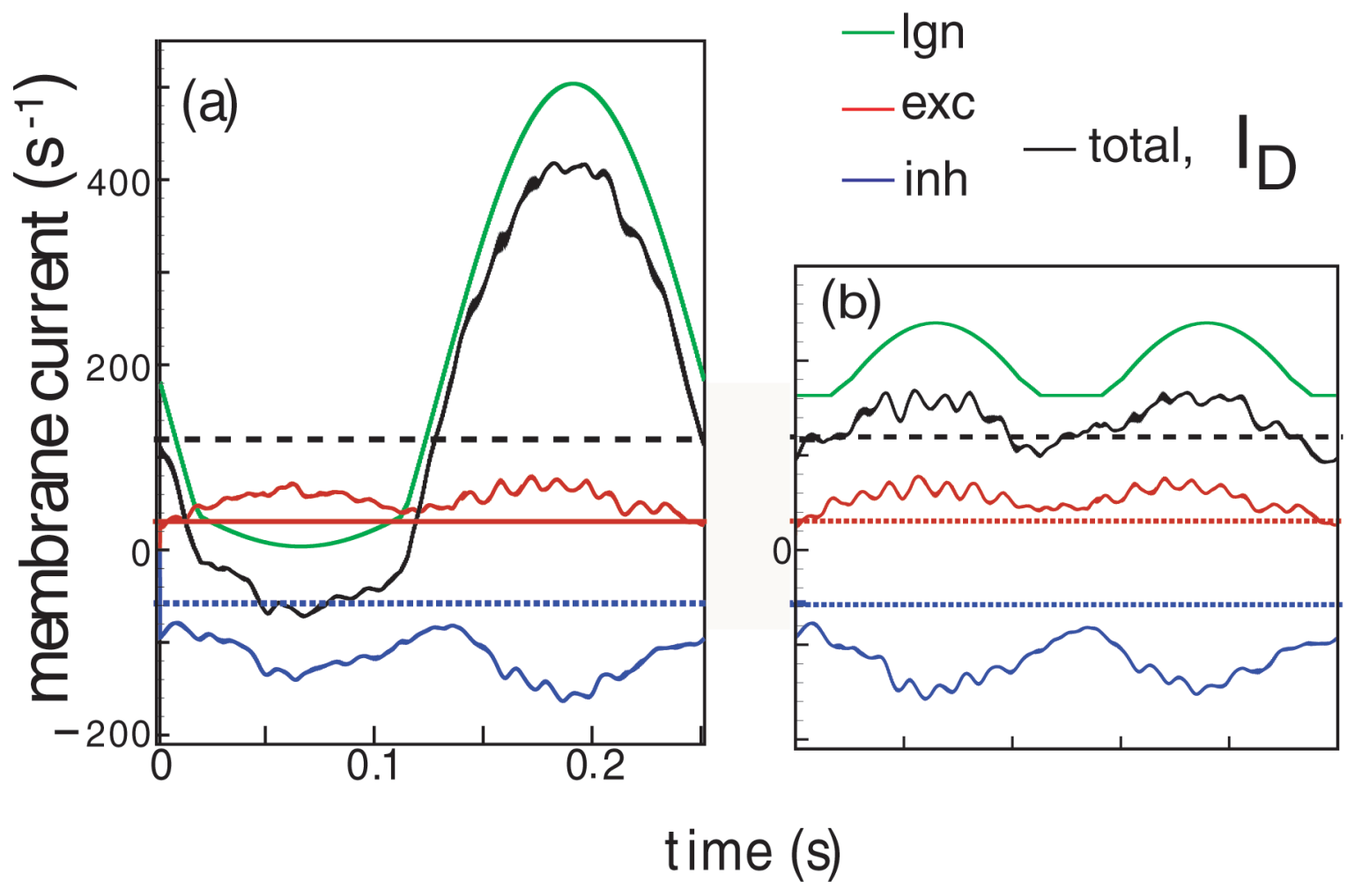

Figure 5. Cycle-averaged currents (averaged over 24 cycles) comprising $\bar{I}_{D}$, for an excitatory neuron in the coupled network, in response to in-phase (a) and orthogonal phase (b) stimulus. Plotted are the LGN ( green), cortical excitatory (red) and cortical inhibitory (blue), and grand total (black) currents. Also plotted are the mean values of the excitatory (red dotted) and inhibitory noises (blue dotted), and total background current (black dotted line).

\section{Drifting grating stimulus}

So far, we have analyzed the response of the model to contrast reversal. We also studied the responses of the model to other stimuli, in particular drifting sine gratings that have also often been used to characterize simple and complex cells (Movshon et al., 1978; Spitzer and Hochstein, 1985; De Valois et al., 1982). As shown in Figure 7, the spike rate and (blocked) membrane potential of a model neuron are modulated approximately sinusoidally when a sine grating at the optimal orientation is drifted over the receptive field of the neuron, as seen in real neurons. As also shown in Figure 7, the total conductance $g_{T}$ is also modulated sinusoidally at the drift rate of the grating but has a large DC offset. The modulation arises primarily from the modulation in the excitatory conductance $g_{E}$, while the offset arises from the inhibitory conductance $g_{I}$, which is essentially unmodulated but elevated over its background value of $180 \mathrm{sec}^{-1}$. The corticocortical contribution to $g_{E}$, like $g_{I}$, is also mostly unmodulated (data not shown). This is consistent with the cortical excitatory and inhibitory conductances of the model in response to contrast reversal, shown in Figure 6. The same phase averaging that produces spatial phase insensitivity of the inhibitory conductance, and current, to contrast reversal also causes no modulation (but an elevated average level) during stimulation with a drifting grating. This is an important consideration in comparing our model with "push-pull" models, as discussed below.

\section{Two predictions of the model}

There are two predictions that are revealing about the mode of action of the model. The first prediction concerns a greater nonlinear temporal modulation expected in corticocortical conductances for neurons farther from pinwheel centers. This is a

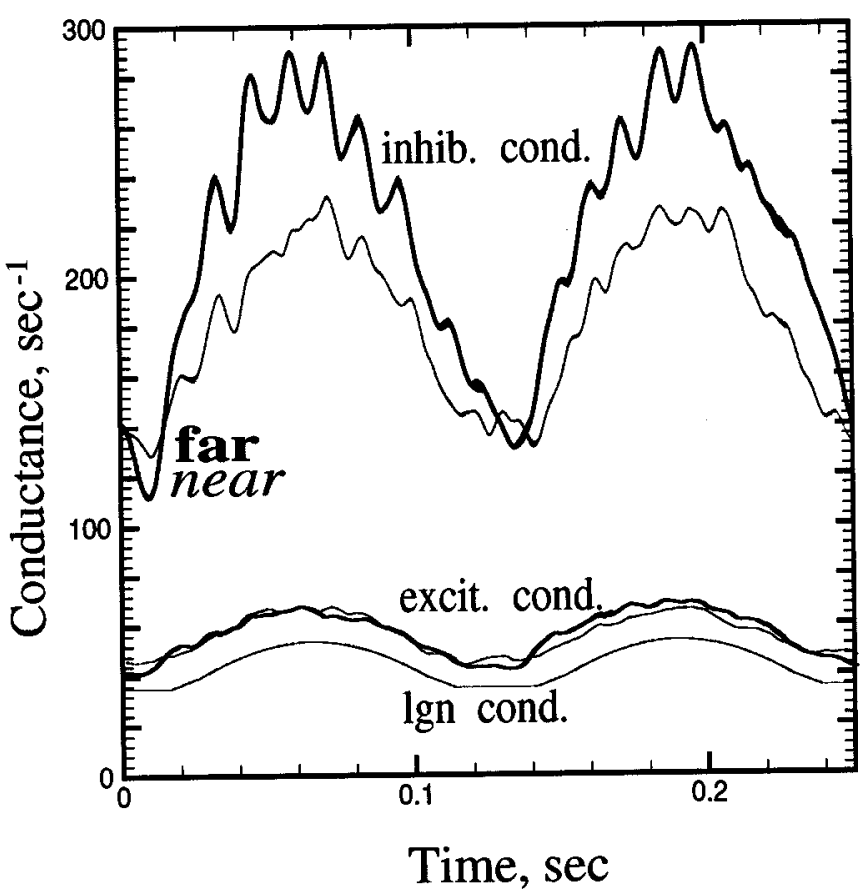

Figure 6. Constituent conductances for the near and far neurons illustrated in this paper, at the orthogonal spatial phase of contrast reversal. The LGN input excitation is the lowest thin curve. Near corticocortical excitation and inhibition are remaining thin curves; far curves are thick. Note the large inhibitory components of the conductances and that the far neuron has significantly larger F2 modulation in its inhibitory conductance than does the near neuron. 


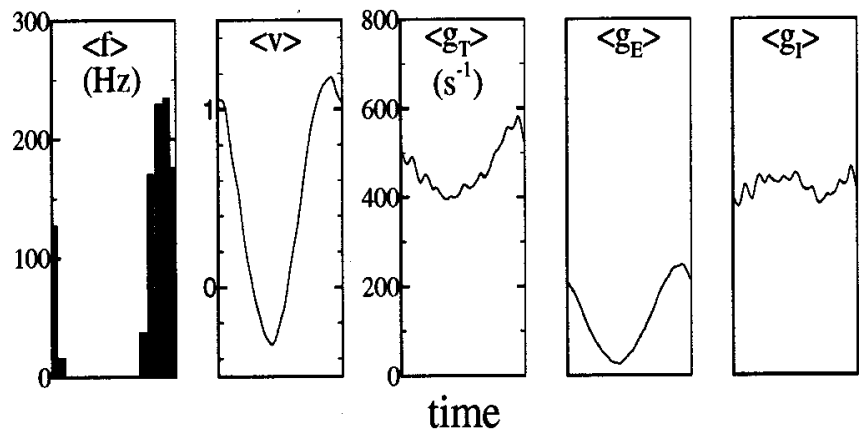

Figure 7. Responses to drifting gratings. This stimulus was a drifting sinusoidal grating at optimal spatial frequency and orientation, at a drift rate of $8 \mathrm{~Hz}$ and $100 \%$ contrast. From left to right, the panels shown are cycle-averaged spike rate, blocked membrane potential, total conductance, excitatory conductance, and inhibitory conductance.

consequence of the lateral coupling having length scales well below the width of the orientation hypercolumn. The second is a prediction that the $F_{2}$ component in the membrane potential of an individual neuron should get larger when the membrane is hyperpolarized with current.

The first prediction is illustrated in Figure 8. These are data from the same network simulation as in Figure 3 but from a different model neuron, one located far from a pinwheel center. These responses should be compared with Figure $3 a-c$ for a neuron near the pinwheel. There is an overall resemblance in the spike rate waveforms between the two neurons in the model, but there is a significant difference in intracellular response: the neuron in Figure 8 has an evident $F_{2}$ component in its membrane potential response at orthogonal phase. The $F_{2}$ component in this cortical cell is opposite in sign from the LGN drive. It is caused by inhibition, which is so strong in this neuron to this stimulus that it overrides the sources of excitation. The model predicts there should be strong inhibition in far neurons, as is evident in Figure 6. In the contrast reversal experiment, only one spatial grating at one orientation is exciting the cortex. This means only one spoke of the pinwheel is being driven hard, most especially by the LGN input near the maximal in-phase case. (Along the spoke at orthogonal preferred angle, the LGN input is relatively phaseinsensitive and of smaller amplitude.) Each neuron in the model receives its inhibitory input predominantly from cortical neurons within a $100 \mu \mathrm{m}$ radius. Far from the pinwheel center, near the spoke that is excited maximally by the orientation of the grating stimulus, this disk of inhibitory input covers many excited neurons
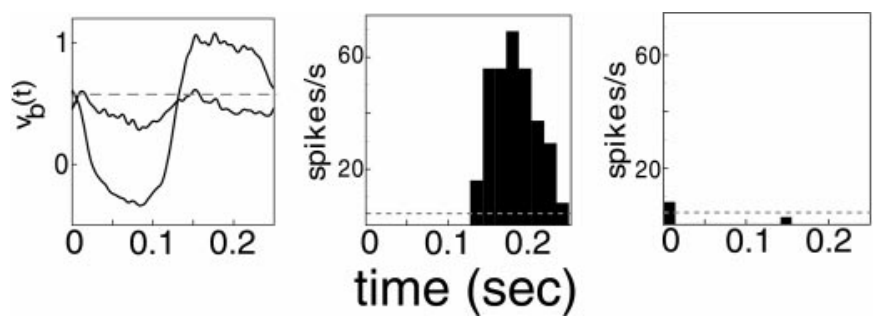

Figure 8. Responses to in-phase and orthogonal phase, $4 \mathrm{~Hz}$ contrast reversal gratings (at 100\% contrast, preferred angle and optimal spatial frequency), for a neuron far from a pinwheel center. The format for this figure is the same as for Figure 3. The cycle-averaged membrane potential is shown, with spike and reset blocked, as in Figure 3, and below it are the cycle-averaged spike rates for in-phase and orthogonal phase stimuli, respectively. The cycle averages were computed for 24 cycles.

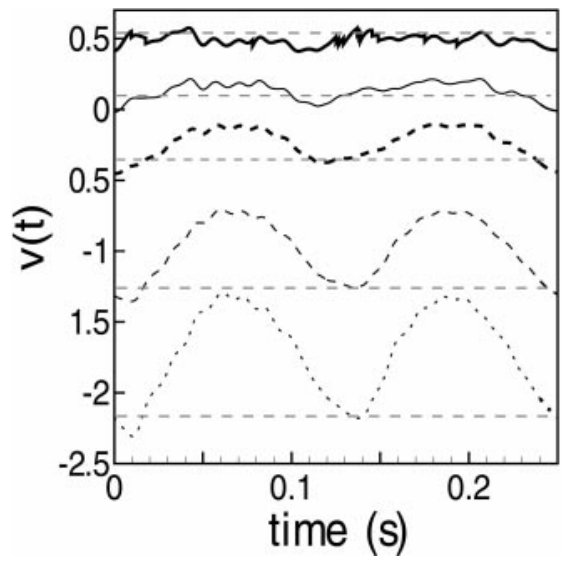

Figure 9. Membrane potential at different holding currents for a neuron near a pinwheel center. The five modulated waveforms are membrane potential responses to sinusoidal contrast reversal in the orthogonal phase condition. These are averaged response waveforms to 24 cycles of stimulus contrast reversal. The top curve is the potential when there is no extrinsic current. The curves below that are, from top to bottom, measured with a constant hyperpolarizing current of $-100,-200,-400,-600$ $\mathrm{sec}^{-1}$. The dashed horizontal lines for each curve are average values of the membrane potential when the uniform background is shown under the same conditions.

of similar orientation preference. But near the pinwheel center, where many preferred orientations are represented, the disk then covers a smaller fraction of neurons well excited by this one orientation, and so the summed inhibition is relatively weaker.

A second prediction concerns changes in the response of a simple cell after injection of a constant transmembrane current. The $F_{2}$ component of the membrane potential of a neuron depends on extrinsic current that polarizes the membrane. Figure 9 shows the membrane potential of the model neuron that was used in Figure 3, at the orthogonal phase, for five different choices of a constant holding current $I_{\text {hold }}$. As this current becomes more negative, there is an increasing temporal modulation at $F_{2}$ that is approximately linear with $I_{\text {hold }}$. Indeed, the subthreshold potential is well described by the large conductance approximation $v \approx$ $I_{D} / g_{T}+I_{\text {hold }} / g_{T}$. For this neuron, $I_{D} / g_{T}$ is approximately constant (Fig. $3 a$ ), whereas $I_{\text {hold }} / g_{T}$ contributes an $F_{2}$ term from $g_{T}$ that grows as $I_{\text {hold }}$ becomes more negative. Similarly, other predictions of the model could be tested experimentally: for values of the holding current for which the potential is subthreshold, one could use linear regression to directly estimate the time-dependent total conductance $g_{T}$ (Borg-Graham et al., 1998; Hirsch et al., 1998), as well as its excitatory and inhibitory components (assuming Eqs. 6 and 7 ). In this way the presence and nature of $F_{2}$ components could be checked experimentally. In particular, by using visual stimuli with different spatial phases, the phase (in)sensitivity of corticocortical inhibition could be examined.

\section{DISCUSSION}

\section{The importance of simple cells}

Our work establishes that the linear behavior of simple cells arises as a consequence of network activity. However, this leads one to ask why does the biological cortical network seem to have this linearity as a goal? The generally accepted view is that, for visual perception, cortical cells must resolve and represent key spatial properties such as surface brightness and color and the perceptual spatial organization of a scene that is the basis of 
form. The existence of simple cells that respond selectively to spatial phase and monotonically to signed contrast are required for the representation of such surface properties and perceptual organization. The large body of work on spatial pattern vision requires linear spatiotemporal neural mechanisms to explain how patterns are detected separately and in combination (for review, see Graham, 1989; Wandell, 1995). Also, theories of color vision implicitly assume the existence of simple cells whenever they postulate the necessity of numerical computations of (signed) edge contrast (for review, see Wandell, 1995). Scene organization requires computation of depth order that in turn depends on computation of stereoscopic depth and also of pictorial occlusion. Both stereo (Anzai et al., 1999a,b) and occlusion (Anderson, 1997) computations require cortical representation of signed edge contrast. Furthermore, the perception of salient contours also has been shown to be sensitive to spatial phase and thus contrast sign (Field et al., 2000). Such neural computations would seem to require the linearity that only simple cells provide. These considerations lead to the conclusion that visual perception needs simple cells for basic functions.

Simple and complex cells were first discovered in cat visual cortex (Hubel and Wiesel, 1962), and their existence confirmed subsequently in macaque V1(Hubel and Wiesel, 1968; De Valois et al., 1982). Simple cells have been found in the primary visual cortex of many other species of mammals: owl monkeys (O'Keefe et al., 1998), baboons (Kennedy et al., 1985), tree shrews (Kaufmann and Somjen, 1979), rats (Burne et al., 1984; Girman et al., 1999), mice (Drager, 1975), rabbits (Glanzman, 1983), and sheep (Kennedy et al., 1983). Their ubiquity in the animal kingdom may be an indicator of their importance.

Neurons in other sensory cortices have linear signal processing properties that resemble those seen in simple cells of the visual cortex. Some neurons in the primary auditory cortex have been characterized as linear transducers of auditory patterns (Kowalski et al., 1996). Similar characterizations in the primary somatosensory cortex have also identified linearly summing neurons with receptive fields similar in many ways to visual simple cells (DiCarlo and Johnson, 2000). The same processes that give rise to the creation of simple cells in visual cortex will be important for understanding how they may be produced elsewhere by the cortical circuitry.

\section{Mechanisms for the production of simple cells}

Our computational model of layer $4 C \alpha$ in macaque $\mathrm{V} 1$ is a nonlinear network of I\&F neurons, driven by LGN input, which is itself nonlinear. Yet, the neurons of the model respond in an approximately linear manner, which is characteristic of simple cells, including (1) a sensitive dependence of the responses of the neurons on spatial phase or position and (2) very little presence of nonlinear distortion (such as second temporal harmonics) in the responses of the neurons. Stimulation by contrast reversal of a standing grating pattern, with the phase of the grating orthogonal to the preferred phase of the simple cell, constitutes a most stringent test of this linearity. Although the temporal profile of the total LGN excitatory drive to each neuron in the network contains significant second harmonic content, the membrane potential of the output of each cell (as measured intracellularly) contains little second harmonic distortion.

Given the nonlinearity of the full network and of the LGN drive, the linear behavior of simple cells is not a simple consequence of feedforward convergence. The active presence of the corticocortical interactions in the network significantly reduces the second harmonic content of the membrane potential of the simple cell, when compared with its temporal waveform in the absence of network interactions. The work reported here has identified the two properties of the network model that are responsible for this linearization of the response: (1) phase averaging of the individual corticocortical inputs to the cell, which collectively produce a frequency-doubled temporal component (as used in Chance et al., 1999); (2) strong inhibition, so that corticocortical inhibitory input tends to cancel the frequencydoubled excitatory input from the LGN. These two properties produce the linear responses of simple cells within the model cortex, and they are likely to be the key network mechanisms that cause the linear behavior of simple cells in the biological cortex. Both phase averaging and strong inhibition are caused by the nature of corticocortical connections in the model.

This model of simple cells as resulting from the cancellation of nonlinear excitation by strong nonlinear inhibition can account for many experiments. It explains why there is a large increase in conductance in a simple cell both at the onset and offset of a flashing bar in the receptive field of a simple cell (Borg-Graham et al., 1996, 1998). As the measurements of Borg-Graham et al. (1996, 1998) indicate, the conductance increase is dominated by the inhibitory conductance, as in our model, and corticocortical inhibition is on-off as in the model. The model also provides a convincing explanation why pharmacological weakening of inhibition would make simple cells appear to be complex (Sillito, 1975; Frégnac and Shulz, 1999; Murthy and Humphrey, 1999), because weakening the inhibition should prevent it from cancelling the nonlinear component of excitation. The data of Murthy and Humphrey (1999) are particularly relevant. They stimulate their simple cells with grating contrast reversal as in our modeling and observe marked frequency doubling in spike rates of simple cells when bicuculline is infused.

Our model is very different from previous attempts to explain cortical linearization in terms of a "push-pull" model (Palmer and Davis, 1981; Tolhurst and Dean, 1990) that requires direct or indirect phase-sensitive inhibition from the LGN. Direct inhibition from LGN to cortical neurons is ruled out by anatomy; LGN-to-cortex inhibitory synapses do not exist. One might attempt to preserve the push-pull concept by postulating that disynaptic inhibition from inhibitory neurons in the cortex could provide phase-sensitive inhibition (as instantiated in the model of Troyer et al., 1998). But then one would have to explain how phase-sensitive inhibition is consistent with the anatomy of inhibitory interneurons in the cortex: such neurons receive many synaptic connections from other cortical cells (Lund, 1987), and there is apparently indiscriminate arborization of axonal branching within the cortex (Fitzpatrick et al., 1985). Nevertheless, previous physiological studies have been interpreted to mean that there is phase-sensitive or push-pull inhibition somehow generated intracortically (Hirsch et al., 1998; Anderson et al., 2000). However, much of this evidence has been indirect.

There is recent evidence on this point (Anderson et al., 2000). From measurements of simple cell responses to drifting gratings, the authors infer that the temporal modulation of synaptic inhibition in opposition to the modulation of synaptic excitation is indicative of push-pull interactions between inhibition and excitation. However, scrutiny of the measurements in Anderson et al. (2000) indicates that there usually is a large phase-insensitive component of the inhibitory conductance, consistent with the phase-insensitive inhibition that is observed in the response of our model to drifting gratings (Fig. 7). Furthermore, the authors 
saw modulation of the measured corticocortical inhibition primarily when the cell was above threshold and firing. It is possible that their measurements of synaptic conductances were made inaccurate by the spiking. Other direct intracellular measurements by Borg-Graham et al. (1998) indicate that inhibition in simple cells is more often spatial phase-insensitive than phase-sensitive (or push-pull), as Borg-Graham et al. (1998) indeed noted. Our model produces unmodulated cortical inhibition in response to drifting gratings because neurons are excited by inhibitory neighbors of different spatial phase preference. In this way our model differs from that of Troyer et al. (1998), whose couplings are explicitly phase-specific. Perhaps the real cortex has inhibitory neurons that are neither wholly phase-insensitive as in our model, nor wholly phase-sensitive as envisioned in push-pull models, but have phase sensitivity somewhere between all-or-none. However, to explain the linearization of cortical simple cell responses and to be consistent with anatomy, our model with spatial-phaseinsensitive cortical inhibition seems closest to the best evidence available now.

\section{REFERENCES}

Anderson BL (1997) A theory of illusory lightness and transparency in monocular and binocular images: the role of contour junctions. Perception 26:419-453.

Anderson JS, Carandini M, Ferster D (2000) Orientation tuning of input conductance, excitation, and inhibition in cat primary visual cortex. J Neurophysiol 84:909-926.

Anzai A, Ohzawa I, Freeman RD (1999a) Neural mechanisms for processing binocular information. I. Simple cells. J Neurophysiol 82:891-908.

Anzai A, Ohzawa I, Freeman RD (1999b) Neural mechanisms for processing binocular information. II. Complex cells. J Neurophysiol 82:909-924.

Azouz R, Gray CM, Nowak LG, McCormick DA (1997) Physiological properties of inhibitory interneurons in cat striate cortex. Cereb Cortex 7:534-545.

Beaulieu C, Kisvarday Z, Somogyi P, Cynader M, Cowey A (1992) Quantitative distribution of GABA immunopositive and immunonegative neurons and synapses in the monkey striate cortex. Cereb Cortex 2:295-309.

Benardete E, Kaplan E (1999) The dynamics of primate M retinal ganglion cells. Vis Neurosci 16:355-368.

Blasdel G (1992a) Differential imaging of ocular dominance and orientation selectivity in monkey striate cortex. J Neurosci 12:3115-3138.

Blasdel G (1992b) Orientation selectivity, preference, and continuity in the monkey striate cortex. J Neurosci 12:3139-3161.

Bonhoeffer T, Grinvald A (1991) Iso orientation domains in cat visual cortex are arranged in pinwheel like patterns. Nature 353:429-431.

Borg-Graham L, Monier C, Fregnac Y (1996) Voltage-clamp measurement of visually-evoked conductances with whole-cell patch recordings in primary visual cortex. J Physiol (Paris) 90:185-188.

Borg-Graham L, Monier C, Fregnac Y (1998) Visual input evokes transient and strong shunting inhibition in visual cortical neurons. Nature 393:369-373.

Burne RA, Parnavelas JG, Lin CS (1984) Response properties of neurons in the visual cortex of the rat. Exp Brain Res 53:374-383.

Callaway E (1998) Local circuits in primary visual cortex of the macaque monkey. Annu Rev Neurosci 21:47-74.

Callaway E, Wiser A (1996) Contributions of individual layer 2 to 5 spiny neurons to local circuits in macaque primary visual cortex. Vis Neurosci 13:907-922.

Chance F, Nelson S, Abbott LF (1999) Complex cells as cortically amplified simple cells. Nat Neurosci 2:277-282.

DeAngelis G, Ghose R, Ohzawa I, Freeman R (1999) Functional microorganization of primary visual cortex: receptive field analysis of nearby neurons. J Neurosci 19:4046-4064.

De Valois R, Albrecht D, Thorell L (1982) Spatial frequency selectivity of cells in macaque visual cortex. Vision Res 22:545-559.

DiCarlo JJ, Johnson KO (2000) Spatial and temporal structure of receptive fields in primate somatosensory area $3 \mathrm{~b}$ : effects of stimulus scanning direction and orientation. J Neurosci 20:495-510.

Drager UC (1975) Receptive fields of single cells and topography in mouse visual cortex. J Comp Neurol 160:269-290.

Enroth-Cugell C, Robson J (1966) The contrast sensitivity of retinal ganglion cells of the cat. J Physiol (Lond) 187:517-552.

Ferster D, Chung S, Wheat H (1996) Orientation selectivity of thalamic input to simple cells of cat visual cortex. Nature 380:249-252.
Field DJ, Hayes A, Hess RF (2000) The roles of polarity and symmetry in the perceptual grouping of contour fragments. Spat Vis 13:51-66.

Fitzpatrick D, Lund J, Blasdel G (1985) Intrinsic connections of macaque striate cortex afferent and efferent connections of lamina 4C. J Neurosci 5:3329-3349.

Frégnac Y, Shulz D (1999) Activity-dependent regulation of receptive field properties of cat area 17 by supervised Hebbian learning. J Neurobiol 41:69-82.

Gibson J, Beierlein M, Connors B (1999) Two networks of electrically coupled inhibitory neurons in neocortex. Nature 402:75-79.

Girman SV, Sauve Y, Lund RD (1999) Receptive field properties of single neurons in rat primary visual cortex. J Neurophysiol 82:301-311.

Glanzman DL (1983) Spatial properties of cells in the rabbit's striate cortex. J Physiol (Lond) 340:535-553.

Graham N (1989) Visual pattern analyzers. Oxford: Oxford UP.

Hirsch J, Alonso JM, Reid R, Martinez L (1998) Synaptic integration in striate cortical simple cells. J Neurosci 15:9517-9528.

Hochstein S, Shapley R (1976) Quantitative analysis of retinal ganglion cell classifications. J Physiol (Lond) 262:237-264.

Hubel D, Wiesel T (1962) Receptive fields, binocular interaction and functional architecture of the cat's visual cortex. J Physiol (Lond) 160:106-154.

Hubel D, Wiesel T (1968) Receptive fields and functional architecture of the monkey striate cortex. J Physiol (Lond) 195:215-243.

Jagadeesh B, Wheat H, Kontsevich L, Tyler C, Ferster D (1997) Direction selectivity of synaptic potentials in simple cells of the cat visual cortex. J Neurophysiol 78:2772-2789.

Kaplan E, Shapley R (1982) X and Y cells in the lateral geniculate nucleus of the macaque monkey. J Physiol (Lond) 330:125-143.

Kaufmann PG, Somjen GG (1979) Receptive fields of neurons in areas 17 and 18 of tree shrews (Tupaia glis). Brain Res Bull 4:319-325.

Kennedy H, Martin KA, Whitteridge D (1983) Receptive field characteristics of neurones in striate cortex of newborn lambs and adult sheep. Neuroscience 10:295-300.

Kennedy H, Martin KA, Whitteridge D (1985) Receptive field properties of neurones in visual area 1 and visual area 2 in the baboon. Neuroscience 14:405-415.

Koch C (1999) Biophysics of computation. Oxford: Oxford UP.

Kowalski N, Depireux DA, Shamma SA (1996) Analysis of dynamic spectra in ferret primary auditory cortex. II. Prediction of unit responses to arbitrary dynamic spectra. J Neurophysiol 76:3524-3534.

Lund JS (1987) Local circuit neurons of macaque monkey striate cortex: Neurons of laminae 4C and 5A. J Comp Neurol 257:60-92.

Maldonado P, Godecke I, Gray C, Bonhoeffer T (1997) Orientation selectivity in pinwheel centers in cat striate cortex. Science 276:1551-1555

McLaughlin D, Shapley R, Shelley M, Wielaard J (2000) A neuronal network model of macaque primary visual cortex (V1): orientation selectivity and dynamics in the input layer $4 C \alpha$. Proc Natl Acad Sci USA 97:8087-8092.

Movshon JA, Thompson ID, Tolhurst DJ (1978) Receptive field organization of complex cells in the cat's striate cortex. J Physiol (Lond) 283:79-99.

Murthy A, Humphrey AL (1999) Inhibitory contributions to spatiotemporal receptive-field structure and direction selectivity in simple cells of cat area 17. J Neurophysiol 81:1212-24.

O'Keefe LP, Levitt JB, Kiper DC, Shapley RM, Movshon JA (1998) Functional organization of owl monkey lateral geniculate nucleus and visual cortex. J Neurophysiol 80:594-609.

Palmer L, Davis T (1981) Receptive-field structure in cat striate cortex. J Neurophysiol 46:260-276.

Reid RC, Alonso J-M (1995) Specificity of monosynaptic connections from thalamus to visual cortex. Nature 378:281-284.

Reid RC, Soodak RE, Shapley RM (1991) Directional selectivity and spatiotemporal structure of receptive fields of simple cells in cat striate cortex. J Neurophysiol 66:505-529.

Ringach DL, Shapley R, Hawken MJ (2001) Diversity and specialization of orientation selectivity in simple and complex cells of macaque V1. $\mathrm{J}$ Neurosci, in press.

Shapley R (1994) Linearity and nonlinearity in cortical receptive fields. In: Higher order processing in the visual system, Ciba Symposium 184, pp 71-87. Chichester, UK: Wiley.

Sillito AM (1975) The contribution of inhibitory mechanisms to the receptive field properties of neurones in the striate cortex of the cat. J Physiol (Lond) 250:305-329.

Spitzer H, Hochstein S (1985) Simple- and complex-cell response dependences on stimulation parameters. J Neurophysiol 53:1244-1265.

Tolhurst D, Dean A (1990) The effects of contrast on the linearity of spatial summation of simple cells in the cat's striate cortex. Exp Brain Res 79:582-588.

Troyer T, Krukowski A, Priebe N, Miller K (1998) Contrast invariant orientation tuning in cat visual cortex with feedforward tuning and correlation based intracortical connectivity. J Neurosci 18:5908-5927.

Wandell BA (1995) Foundations of vision. Sunderland, MA: Sinauer. 